\title{
Venous Thromboembolism Complicated with COVID-19: What Do We Know So Far?
}

\author{
Shir Tal ${ }^{a, c}$ Galia Spectre ${ }^{b, c}$ Ran Kornowski ${ }^{a, c}$ Leor Perl ${ }^{a, c}$ \\ ${ }^{a}$ Cardiology Department, Rabin Medical Center, Petah Tikva, Israel; ${ }^{b}$ Hematology Department, Rabin Medical \\ Center, Petah Tikva, Israel; ' Sackler Faculty of Medicine, Tel Aviv University, Tel Aviv, Israel
}

\section{Keywords}

Venous thromboembolism · Pulmonary emboli · Deep vein thrombosis - COVID-19

\begin{abstract}
Coronavirus disease (COVID-19) is caused by the novel severe acute respiratory syndrome coronavirus 2 (SARS-CoV-2) and is responsible for the ongoing 2019-2020 pandemic. Venous thromboembolism (VTE), a frequent cardiovascular and/or respiratory complication among hospitalized patients, is one of the known sequelae of the illness. Hospitalized COVID-19 patients are often elderly, immobile, and show signs of coagulopathy. Therefore, it is reasonable to assume a high incidence of VTE among these patients. Presently, the incidence of VTE is estimated at around $25 \%$ of patients hospitalized in the intensive care unit for COVID-19 even under anticoagulant treatment at prophylactic doses. In this review, we discuss present knowledge of the topic, the unique challenges of diagnosis and treatment of VTE, as well as some of the potential mechanisms of increased risk for VTE during the illness. Understanding the true impact of VTE on patients with COVID-19 will potentially improve our ability to reach a timely diagnosis and initiate proper treatment, mitigating the risk for this susceptible population during a complicated disease.

(c) 2020 S. Karger AG, Basel
\end{abstract}

\section{Introduction}

Since December 8, 2019, a cluster of cases of "pneumonia of unknown origin" in humans, associated with the Huanan Seafood Wholesale Market, has been reported in Wuhan, China. The disease, which is the third coronavirus infection [1], is caused by the severe acute respiratory syndrome coronavirus 2 (SARS-CoV-2) and was named coronavirus disease 2019 (COVID-19) by WHO [2].

Up to April 17, 2020, over 2 million patients were infected and more than 135,000 died in 213 countries [3].

Upon initial recognition, patients can be asymptomatic carriers or have a range of symptoms with a presentation resembling pneumonia, showing mainly fever, cough, fatigue, and dyspnea [4-7]. Several recent studies suggest a hypercoagulable state in patients presenting with COVID-19. Laboratory findings show high CRP, leukopenia, lymphocytopenia, mild thrombocytopenia, prolonged PT, high D-dimers, and high fibrinogen levels early in the disease course, which may be complicated by low fibrinogen later on in severe cases [8-10]. Imaging shows bilateral pneumonia in about $75 \%$ of patients $[5$, 6], but can also show other patterns such as ground glass opacities, patchy shadowing, and consolidation $[6,9]$. The diagnosis is confirmed with a real-time protein chain reaction (RT-PCR) assay $[5,6,9]$.

karger@karger.com
www.karger.com/aha
Karger $V^{\prime /}$


The differential diagnosis is wide. Respiratory infections such as influenza and other flu-like upper respiratory viral diseases, bacterial pneumonia, and COPD exacerbation all commonly present during winter time, coinciding with the emergence of the pandemic. In addition, cardiovascular morbidity such as heart failure and venous thromboembolism (VTE) may lead to respiratory distress symptoms resembling COVID-19.

VTE is the third most frequent acute cardiovascular disease, following myocardial infarction and stroke, with an annual incidence rate of 39-115 per 100,000 population for pulmonary embolism (PE) and an incidence rate of 53-162 per 100,000 population for deep vein thrombosis (DVT) [11]. It is the cause of over 100,000 deaths annually and is considered the most preventable cause of death in hospitalized patients in the USA [12]. There is a somewhat higher incidence of VTE among hospitalized patients, which is more in high-income countries compared to low- and middle-income countries (3.3 vs. $3 \%$ ) [13].

Many of the hospitalized COVID-19 are elderly patients who suffer from severe infectious illness, some immobile in an intensive care unit (ICU) setting. Therefore, a relatively high incidence of VTE among patients diagnosed with COVID-19 is expected, due to the severity of their disease and distinctive risk factors.

The aims of this review are to present the current knowledge of the unique challenge of diagnosis and treatment of VTE as well as some of the potential mechanisms that link VTE to patients with COVID-19.

\section{Risk of VTE in COVID-19}

Patients with COVID-19 have a relatively prolonged disease, with a duration that ranges between 17 and 25 days. [5] Although most patients have a favorable prognosis, older patients and those with chronic underlying conditions may have worse outcomes. At the writing of this paper (April 17, 2020), the average death rate worldwide is about $6.6 \%$ [3]. About one third of the worsened patients [5, 6] and up to $41.8 \%$ [7] suffer from complications such as respiratory failure, acute respiratory distress syndrome (ARDS). In addition, they may have heart failure, secondary bacterial infections, and septic shock.

During their illness the patients may require high-flow oxygen, inhalations, vasopressors, mechanical ventilations, and even ECMO. The complexity of disease in many patients necessitates a large number of highly qualified medical personnel to deal with these unique challenges.

\section{Incidence of VTE in Patients with COVID-19}

Patients with COVID-19 infection are at high risk of developing thromboembolic complications. However, so far there have been only two studies describing VTE: DVT or PE in patients with COVID-19, as well as several case reports. Klok et al. [14] reported a high incidence of VTE in critically ill patients admitted to the ICU despite the use of at least prophylactic anticoagulation. They evaluated $184 \mathrm{ICU}$ patients - mean age $64 \pm 12$ years, 139 (76\%) of which were male, from 2 Dutch university hospitals and 1 Dutch teaching hospital, positive to COVID-19. Of these patients, 23 died (13\%), 22 were discharged alive (12\%), and $139(76 \%)$ were still in the ICU on April 5, 2020. Computer tomography pulmonary angiogram (CTPA) and/or ultrasonography done by clinical suspicion confirmed VTE in $27 \%$ (95\% CI 17-37\%) and arterial thrombotic events in 3.7\% (95\% CI, 0-8.2\%). $\mathrm{PE}$ was the most frequent thrombotic complication $(n=$ $25,81 \%$ ). Age (adjusted hazard ratio 1.05/year, 95\% CI $1.004-1.01$ ) and coagulopathy, defined as spontaneous prolongation of the prothrombin time $>3 \mathrm{~s}$ or activated partial thromboplastin time $>5 \mathrm{~s}$ (adjusted hazard ratio $4.1,95 \%$ CI 1.9-9.1), were independent predictors of thrombotic complications. None of the patients developed disseminated intravascular coagulation. In a small study describing autopsies from Brazil, 8/10 patients who died from COVID-19 had pulmonary microthrombi [15].

Cui et al. [8] described the course of 81 patients diagnosed with COVID-19 pneumonia in the ICU of Tongji Medical College, Huazhong University of Science and Technology. Their mean age was 59.9 years (range $32-91$ years) and 37 (46\%) were male; $64(79 \%)$ patients have been discharged from the hospital, $8(10 \%)$ had died, and the rest $9(11 \%)$ remained hospitalized. No preventive anticoagulation was administered; $20 / 81$ patients $(25 \%)$ developed lower-extremity venous thrombosis. The rate of $\mathrm{PE}$ or of ruled-out PE was not mentioned in the article. However, patients with DVT were confirmed, and these were older (68.4 \pm 9.1 vs. $57.1 \pm 14.3$ years, $p<0.001)$, had lower lymphocyte counts $\left(0.8 \pm 0.4\right.$ vs $1.3 \pm 0.6 \times 10^{9} / \mathrm{L}$, $p<0.001)$, longer APTT $(39.9 \pm 6.4$ vs. $35.6 \pm 4.5 \mathrm{~s}, p=$ $0.001)$, and higher D-dimer ( $5.2 \pm 3.0$ vs. $0.8 \pm 1.2 \mu \mathrm{g} / \mathrm{mL}$, $p<0.001$ ). Cui et al. [8] also showed that if a cut-off value of $1.5 \mu \mathrm{g} / \mathrm{mL} \mathrm{D}$-dimer was used to predict VTE, the sensitivity was $85.0 \%$, the specificity was $88.5 \%$, and the negative predictive value was $94.7 \%$

In contradictory fashion, Yao et al. [16] from Renmin Hospital of Wuhan University (Wuhan, China) reported 
that $\mathrm{D}$-dimer elevation $(\geq 0.50 \mathrm{mg} / \mathrm{L})$ upon admission was present in $74.6 \%(185 / 248)$ of patients with COVID-19 in whom VTE was theoretically ruled out. However, ruling out VTE in their trial was based mainly on the Wells score, while Doppler ultrasound and CTPA was performed in only 4 patients with a high clinical suspicion for VTE. In the report, D-dimer was associated with both increased disease severity and in-hospital mortality. A Ddimer level of $>2.14 \mathrm{mg} / \mathrm{L}$ predicted in-hospital mortality with a sensitivity of $88.2 \%$ and specificity of $71.3 \%$.

Danzi et al. [17] described a 75-year-old COVID19-positive female patient hospitalized with bilateral pneumonia who was diagnosed with intermediate-/highrisk PE who was hemodynamically stable. She had no predisposing factors other than the acute infection with COVID-19. The diagnosis was confirmed with CTPA showing bilateral filling defect. Echocardiography demonstrated a dilated and severely hypokinetic right ventricle (RV) with a mean derived pulmonary arterial pressure of $60 \mathrm{~mm} \mathrm{Hg}$. She had high CRP $(180 \mathrm{mg} / \mathrm{L})$, troponin I $(3,240.4 \mathrm{ng} / \mathrm{mL})$, and D-dimer $(21 \mu \mathrm{g} / \mathrm{mL})$. Lower limb compression ultrasonography was negative. She was treated with low-molecular-weight heparin (LMWH), lopinavir/ritonavir, and hydroxychloroquine.

Xie et al. [18] reported 2 cases from Wuhan, China, of a 57 -year-old male and a 70-year-old male. Both had pneumonia positive to COVID-19 with fever, cough, dyspnea, and bilateral ground-glass opacities on CT at admission on day 10 and 7 of symptoms, respectively. Due to respiratory deterioration and high $\mathrm{D}$-dimer both went through CTPA which confirmed the diagnosis of PE, on day 2 and 6 of admission, respectively.

\section{Difficulties in the Diagnosis of VTE in Patients with COVID-19}

Patients presenting with COVID-19 typically have a long course of disease, lasting up to several weeks [5]. Some will need high oxygen supply and others will be intubated or treated with vasopressors. During this extended period of time, signs, symptoms and laboratory tests pointing to the diagnosis of VTE can be masked and attributed to COVID-19 or other complications occurring in the prolonged and sometimes complex hospitalization. D-dimer is increased in most of the patients, and usually cannot eliminate the presence of VTE. Therefore, timely diagnosis of VTE is anticipated to pose a significant challenge.

The clinical signs and symptoms of acute PE, the most menacing VTE event, are nonspecific $[11,12]$. Some of the patients are asymptomatic and others have dyspnea, chest pain, hemoptysis, presyncope or syncope, and up to hemodynamic instability. They can overlap with the symptoms of COVID-19 infection or its associated complications, such as ARDS, pleural effusion, or myocarditis [4-7].

Having one or more of the predisposing factors for VTE can be a clue to the diagnosis that otherwise can be missed. COVID-19 patients may have such factors at baseline or acquire them during their illness and hospitalization - older age, elevated CRP, D-dimer, fibrinogen levels, tachypnea, fever, critical illness, infectious etiology, and immobility $[11-13,19]$. Risk scores such as the Wells score or the Geneva clinical prediction score may also be useful, sparing unnecessary tests when predicting low risk for VTE. Hypoxemia, ECG changes indicating RV strain, sinus tachycardia, or atrial fibrillation may be present but they are not specific for PE and can be attributed to other complications of COVID-19.

The diagnostic approach of $\mathrm{PE}[11,12]$ is different between unstable and stable patients. The workup of hemodynamically unstable patients requires CTPA which is the method of choice for imaging. Mobilizing critically ill patients outside of the ICU for imaging may be burdensome and puts both the patients and their surroundings at additional risk of contamination.

Transthoracic echo (TTE) and troponin levels are also part of the workup of unstable patients. TTE may indicate RV overload and/or dysfunction or even a right-sided thrombus. Nevertheless, elevated troponin is present in many of the patients positive to COVID-19 and indicates a severe infection $[5,20]$. In many cases it is a marker of myocardial injury among these patients $[21,22]$. Therefore, in the COVID-19 patient, an abnormal echocardiography test or high troponin levels can also be attributed to the severe pulmonary disease, the "cytokine storm" associated with the viremia, and its systemic effects. It is therefore crucial to note that while both TTE and troponin levels can assist in reaching the diagnosis of $\mathrm{PE}$, one needs a high level of suspicion in order to actively search for VTE and perform the confirmatory tests.

Stable patients with suspected PE can be initially evaluated with a D-dimer test if they are at low or intermediate clinical probability for VTE. However, many of the COVID-19 patients may present with high levels of $\mathrm{D}$-dimer due to other causes - inflammation, disseminated intravascular coagulation, advanced age, or infection [23] suggesting the need for CTPA as an initial rule-out test as well. Nonetheless, as suggested by $\mathrm{Yu}$ et al. [24], who showed that D-dimer levels were significantly correlated 
with inflammation, in cases where the inflammatory state subsides, when the levels of D-dimer are nonproportionally high, VTE should be considered.

V/Q scintigraphy (ventilation/perfusion lung scan) is also a feasible part of the workup in stable cooperative patients with suspected PE. However, this test exposes nuclear medicine workers to aerosolized secretions due to leakage of the aerosol to the room and because the patients frequently cough after inhalation. Using a N-95 mask may reduce the risk of infection, and it is possible to perform perfusion scans only (without the ventilation part), but choosing CTPA as the foremost imaging modality may be a better option [25].

Finally, compression ultrasonography shows DVT only in $30-50 \%$ of patients with PE, but has a sensitivity of $>90 \%$ and a specificity of $\geq 95 \%$ for proximal symptomatic DVT [26], in which case it is a class I indication for the diagnosis of PE [11]. This test must therefore be considered in patients with COVID-19 as well, in appropriate cases.

As for the diagnosis of DVT in COVID-19 patients, a low $\mathrm{D}$-dimer level may help in ruling out the diagnosis. Nevertheless, most patients cannot be regarded as low probability risk, and if clinically suspected, compression ultrasonography is the method of choice, starting from proximal ultrasound and if negative performing wholeleg ultrasound with a sensitivity of $96-99 \%$ and specificity of $99.8 \%$ for lower-extremity DVT [27].

COVID-19 is very contagious with an average reproduction number $\left(R_{0}\right)$ of 3.28 [28], which indicates the transmissibility of the virus by an infectious person in a totally naive population. This complicates the workup of patients, when compared with other patients who are not infected. Every single physical examination, laboratory test, or imaging examination of the patients, including CTPA or echocardiography, requires the full protection of the staff. In addition, all machinery must go through sterilization after use - a process which is time-consuming and may add hardship to what is an already strained health care system. This makes every contact with the patients bothersome, as well as precarious, for both the medical team and other patients within the medical facility.

\section{Treatment of VTE Patients with COVID-19}

Treatment of patients suffering from $\mathrm{PE}[11,12,29]$, in a similar fashion to the diagnosis of $\mathrm{PE}$, is also different in unstable and stable patients. In the unstable patient, reperfusion therapy by thrombolysis and unfractionated heparin (UFH) is the treatment of choice. Nevertheless, many of the patients with COVID-19 have an absolute or a relative contraindication to thrombolysis, such as coagulopathy, thrombocytopenia, a recent invasive procedure, pericarditis, and age $>75$ years $[5,6,30]$. Moreover, even those who are able to receive thrombolysis may require further invasive diagnostics or interventions regarding their COVID-19 infection which thrombolysis may prevent (such as insertion of central-line, pericardiocentesis, insertion of a chest tube, or performing ECMO). It may be difficult to differentiate, but one also needs to keep in mind that if the hemodynamic instability is due to the infectious disease and not to VTE, the treatment of choice is not thrombolysis. One of the indications for PE as a cause for hemodynamic instability is the function of the RV. RV failure related to PE may also respond to a fluid challenge or to vasopressors, both of which may already be administered for some of the COVID-19 patients. No cases of thrombolytic therapy for PE in COVID-19 patients have been published so far. However, thrombolysis was used off-label for acute ARDS [28, 31].

The anticoagulation therapy of stable PE patients is usually LMWH or direct oral anticoagulants (DOACs). In patients with intermediate-/high-risk PE, UFH may be preferred due to its short half-life and the opportunity to give the patients protamine sulfate as an antidote in case of the need for an urgent procedure or bleeding. On the other hand, UFH requires close monitoring which we may want to avoid in such a contagious disease. Therefore, in COVID-19 patients, LMWH may be preferred. For practical reasons, some institutions will prefer DOACs in order to reduce the contact between the nurses and COVID-19 patients [32]. However, drug interaction between DOACs and medical treatment for COVID-19 infection should be considered, as well as the potential risk of organ deterioration in these patients and lack of an effective reversal agent in some centers [33, 34]. Inferior vena cava filters should be considered in selected patients with acute PE and absolute contraindications to anticoagulation, or in case of recurrence despite proper anticoagulant treatment [11]. Graduated compression stockings are no longer recommended for the treatment of DVT [29].

As for the timing of initiation of therapy, the recent European Society of Cardiology (ESC) PE guidelines [11] emphasize the importance of induction of anticoagulation therapy in all patients suspected for PE with high or intermediate clinical probability of $\mathrm{PE}$ without delay, while diagnostic workup is in progress. These guidelines 


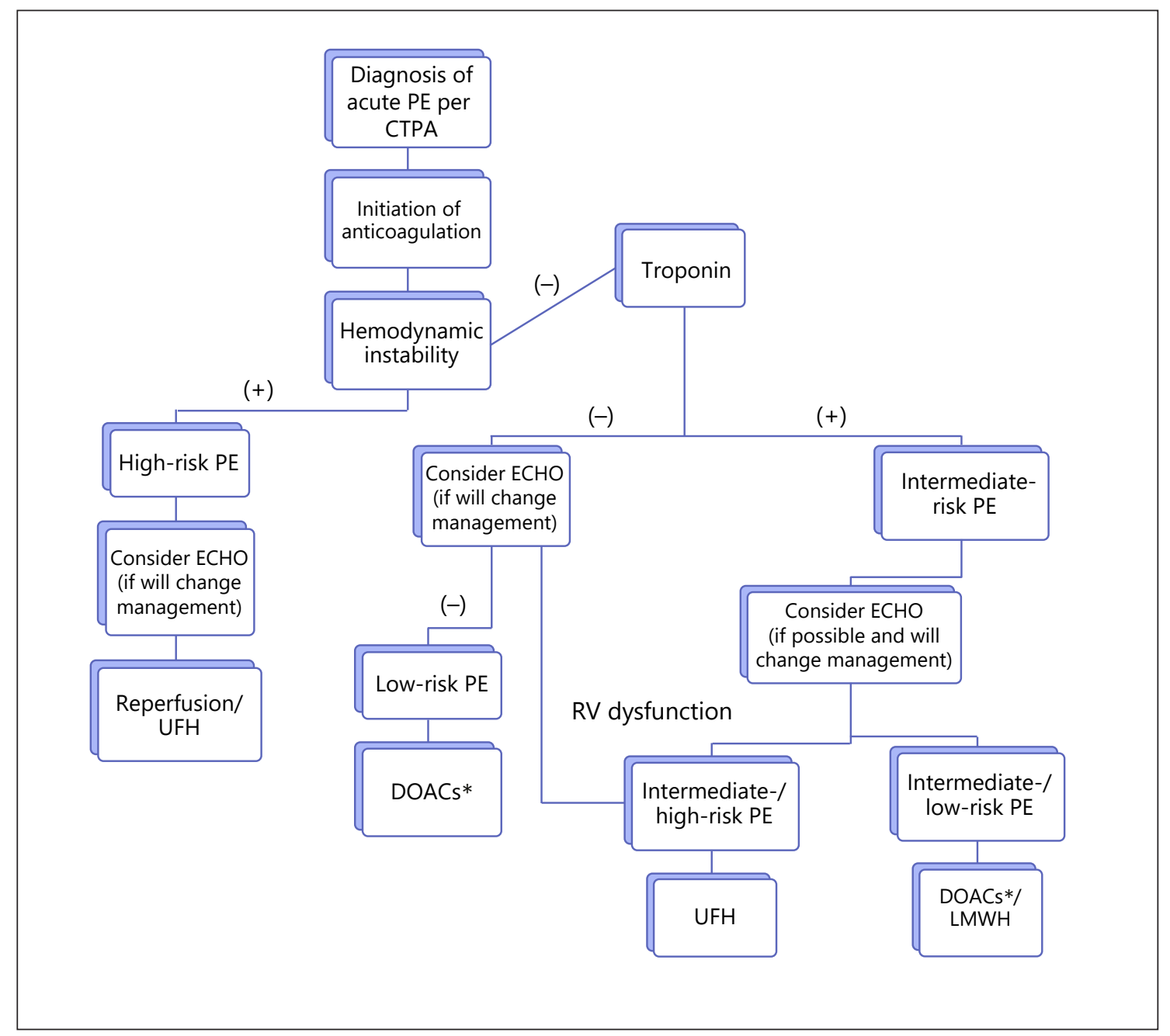

Fig. 1. Pulmonary embolism (PE) management algorithm in the COVID-19 patient. Diagnostic and treatment algorithm of acute PE among COVID-19 patients. CTPA, computer tomography pulmonary angiogram; DOACs, direct oral anticoagulants; LMWH, low molecular weight heparin; UFH, unfractionated heparin. * May be controversial. Please note drug-drug interactions.

are most relevant in COVID-19 patients due to the very possible delay in diagnosis on account of protection of the medical team, sterilization of the machinery, and the difficulty in transporting hemodynamically unstable or intubated patients out of the ICU. As previously mentioned, patients with COVID-19 may present variably, from asymptomatic carriers up to a severe disease requiring admission to the ICU [4-6]. Therefore, the treatment of VTE in these patients must be tailored personally, according to their infectious condition, VTE severity, and general status [12].

Figure 1 presents our suggestion for PE management algorithm in the COVID-19 patient which combines all the abovementioned factors.

VTE Complicated with COVID-19: What Do We Know So Far?

\section{Thrombophylaxis}

A retrospective analysis of 449 COVID patients and 104 non-COVID patients in Tongji Hospital showed that prophylactic anticoagulation therapy (40-60 mg enoxaparin per day or UFH 10,000-15,000 U/day) can reduce mortality, compared to those who do not receive it, only in the COVID group [35].

Another trial of the same group [36] showed that heparin treatment for 7 days in 99 out of 449 patients with COVID-19 reduced 28-day mortality only in patients with a sepsis-induced coagulopathy score $\geq 4$ (40\% vs. $64.2 \%, p=0.029$ ) or patients with $\mathrm{D}$-dimer $>6$-fold of the upper limit of normal (32.8 vs. $54 \% p=0.017$ ).

Acta Haematol 2020;143:417-424 
In the study by Klok et al. [14], all patients hospitalized in the ICU received at least standard thromboprophylaxis doses. We may assume that the rate of VTE without prophylaxis would have been much higher.

Guidelines from Britain recommend VTE prophylaxis with LMWH for all high-risk patients as well as considering the search for PE in patients with sudden onset of oxygenation deterioration, respiratory distress, and reduced blood pressure [32].

Finally, based on the correlation between high levels of D-dimer and severe COVID-19 disease $[7,37]$ as well as higher mortality rate $[5,38]$, the International Society on Thrombosis and Haemosthasis (ISTH) and American Society of Hematology (ASH) guidelines [39] advises prophylactic LMWH in all hospitalized COVID-19 patients in the absence of any contraindications (active bleeding and platelet count less than $25 \times 10^{9} / \mathrm{L}$ ) [34, 40].

In view of the high thromboembolic risk in patients with COVID-19 despite anticoagulation treatment [14], and in view of the thrombotic coagulopathy and extremely high D-dimers with no evidence of clinical bleeding in COVID-19 patients, some institutes like our own recommend considering higher prophylactic doses of anticoagulation such as enoxaparin $0.5 \mathrm{mg} / \mathrm{kg}$ b.i.d. or enoxaparin $1 \mathrm{mg} / \mathrm{kg}$ once daily.

\section{Potential Mechanisms of Increased VTE Risk in Patients with COVID-19}

As in other critically ill patients, patients with COVID-19 fulfill at least two out of three criteria of Virchow's triad - reduced venous flow from immobility and prothrombotic changes due to inflammatory state [41]. Prone position, being used in some of the COVID-19 patients, placing the heart at a hydrostatic level above the head and four extremities, may explain some of the reduction in venous return $[42,43]$. There is evidence to suggest that the third criteria of Virchow's triad - vessel wall changes - may also exist in patients with COVID-19. As was previously reported, serum levels of angiotensin 2 are significantly elevated in COVID-19 patients, activating the renin-angiotensin system, which may cause widespread endothelial dysfunction [44]. In addition, the virus can bind to the endothelial cells via angiotensin 2 receptors, which are most commonly found in the alveolar epithelial cells, followed by endothelial cells - a process which may ultimately damage blood vessels and increase the risk of thrombogenicity $[32,45]$.
As previously published in the literature [11], diagnosis of VTE is quite common in hospitalized patients, and in particular in patients with severe systemic infection. A previous retrospective trial of 18,928 pneumococcal pneumonia patients and 75,712 controls showed that there was higher risk of developing DVT and PE (1.78fold and 1.97-fold, respectively) in patients with pneumococcal pneumonia compared to controls [46]. This suggests a causal with infectious etiology and eludes to the fact that COVID-19 patients might also have a higher incidence of VTE due to their inflammatory condition.

In addition, antiphospholipid antibodies that can arise transiently in patients with critical illness and various infections may possibly develop among COVID-19 patients as well. A case report [47] of critically ill patients with confirmed COVID-19 from Wuhan, China, described 3 patients with clinically significant coagulopathy - ischemia of the lower limbs, multiple cerebral infracts, and antiphospholipid antibodies (anticardiolipin IgA, anti$\beta_{2}$-glycoprotein I IgA, and IgG). Two of the patients, a 69-year-old male and a 65-year-old female, had thrombocytopenia with 78,000 and 79,000 platelets, respectively. The former also had high troponin $(3876.8 \mathrm{pg} / \mathrm{mL})$ and D-dimer levels (>21 mg/L).

Another possible mechanism is activation of the complement system. This theory is based on evidence from studies in murine models investigating complement activation by coronavirus infections, SARS and Middle East respiratory syndrome (MERS), showing laboratory markers consistent with excessive complement activation: elevated lactic dehydrogenase, $\mathrm{D}$-dimer, and bilirubin in addition to decreased platelets, mild anemia, and renal and cardiac injury such as in diffuse thrombotic microangiopathy and atypical hemolytic uremic syndrome [48].

One more final possible mechanism for the high coagulability in COVID-19 patients is hypoxia, as observational and experimental studies show that conditions of hypoxia are associated with increased risk of thrombosis [49].

\section{Conclusion}

VTE is a common entity in hospitalized patients [13] and a preventable cause of death [12]. In patients with COVID-19 VTE poses a unique challenge. First, one needs to suspect the diagnosis despite the wide differential diagnosis of respiratory entities and causes of hemodynamic deterioration in these patients, such as second- 
ary bacterial infection, ARDS, heart failure, and septic shock. Subsequently, if the suspicion is high enough, one needs to consider initiating full-dose anticoagulation and the risk accompanied by the diagnostic workup, in terms of the chance of contamination and the mobilization of the hemodynamic/respiratory unstable patient out of the ICU.

As is expected in the midst of a pandemic breakthrough, there is presently paucity of data regarding the true incidence and characteristics of VTE among COVID-19-positive patients. In fact, there are presently only two trials $[8,14]$ describing an incidence of $27 \%$ for VTE in patients hospitalized in the ICU receiving prophylactic anticoagulation and 25\% for DVT in patients hospitalized in the ICU for COVID-19 (with no mention of the incidence of PE), as well as a few case reports [17, 18]. More information will certainly be published in the near future.

Although COVID-19 is a prolonged, complicated, and sometimes fatal disease, most of the patients are expected to eventually recover. Nonetheless, during the admission, several complications may occur which will impact the prognosis of these patients. Such is the case with VTE, especially due to the risk of underdiagnosis and under- treatment. Thus, the importance in understanding the actual burden of VTE among these patients, and the proper treatment algorithms under these unique circumstances, cannot be overemphasized. A validated treatment for COVID-19 does not exist yet, but VTE is a well-established medical entity, with potentially preventable and treatable consequences.

\section{Disclosure Statement}

The authors have no conflict of interest to declare.

\section{Funding Sources}

The authors have no funding sources to declare.

\section{Author Contributions}

All authors contributed to the study design and revised the manuscript which was drafted by Dr. Tal and Dr. Perl. All authors approved the final version submitted for publication.

\section{References}

1 Morens DM, Daszak P, Taubenberger JK. Escaping Pandora's Box - Another Novel Coronavirus. N Engl J Med. 2020 Apr;382(14): 1293-5.

2 Hui DS, I Azhar E, Madani TA, Ntoumi F, Kock R, Dar O, et al. The continuing 2019$\mathrm{nCoV}$ epidemic threat of novel coronaviruses to global health - The latest 2019 novel coronavirus outbreak in Wuhan, China. Int J Infect Dis. 2020 Feb;91:264-6.

3 World Health organization. Coronavirus disease (COVID-19) pandemic [cited $2020 \mathrm{Apr}$ 7]. Available from: https://www.who.int.

4 Yang J, Zheng Y, Gou X, et al. Prevalence of comorbidities and its effects in patients infected with SARS-CoV-2: a systematic review and meta-analysis. Int J Infect Dis. 2020 Mar; 94:91-5. Available from: https://doi. org/10.1016/j.ijid.2020.03.017.

5 Zhou F, Yu T, Du R, Fan G, Liu Y, Liu Z, et al. Clinical course and risk factors for mortality of adult inpatients with COVID-19 in Wuhan, China: a retrospective cohort study. Lancet. 2020 Mar;395(10229):1054-62.

6 Chen N, Zhou M, Dong X, Qu J, Gong F, Han $\mathrm{Y}$, et al. Epidemiological and clinical characteristics of 99 cases of 2019 novel coronavirus pneumonia in Wuhan, China: a descriptive study. Lancet. 2020 Feb;395(10223):507-13.

7 Wu C, Chen X, Cai Y, Xia J, Zhou X, Xu S, et al. Risk factors associated with acute respiratory distress syndrome and death in patients with coronavirus disease 2019 pneumonia in Wuhan, China. JAMA Intern Med. 2020. Available from: https://doi.org/10.1001/jamainternmed.2020.0994.

8 Cui S, Chen S, Li X, Liu S, Wang F. Prevalence of venous thromboembolism in patients with severe novel coronavirus pneumonia. J Thromb Haemost. 2020. doi:https://doi. org/10.1111/jth.14830.

9 Guan W, Ni Z, Hu Y, et al. Clinical characteristics of coronavirus disease 2019 in China. $\mathrm{N}$ Engl J Med. 2020;382:1708-20. Available from: $\quad$ https://doi.org/10.1056/NEJMoa2002032.

10 Tang N, Li D, Wang X, Sun Z. Abnormal coagulation parameters are associated with poor prognosis in patients with novel coronavirus pneumonia. J Thromb Haemost. 2020 Apr; 18(4):844-7.

11 European Society of Cardiology. 2019 ESC Guidelines for the diagnosis and management of acute pulmonary embolism developed in collaboration with the European Respiratory Society (ERS): The Task Force for the diagnosis and management of acute pulmonary embolism of the European Society of Cardiology (ESC) [cited 2020 Apr 7]. Available from: https://academic.oup.com/eurheartj/article/41/4/543/5556136

12 Vikas Aggarwal V, Nicolais CD, Lee A, Bashir R. Acute management of pulmonary embolism [cited 2020 Apr 14]. Available from: https://www.acc.org/latest-in-cardiology/articles/2017/10/23/12/12/acute-managementof-pulmonary-embolism.

13 Raskob GE, Angchaisuksiri P, Blanco AN, Buller H, Gallus A, Hunt BJ, et al.; ISTH Steering Committee for World Thrombosis Day. Thrombosis: a major contributor to global disease burden. Arterioscler Thromb Vasc Biol. 2014 Nov;34(11):2363-71.

14 Klok FA, Kruip MJ, van der Meer NJ, Arbous MS, Gommers DA, Kant KM, et al. Incidence of thrombotic complications in critically ill ICU patients with COVID-19. Thromb Res. 2020 Apr:S0049-3848(20)30120-1.

15 Dolhnikoff M, Duarte-Neto AN, de Almeida Monteiro RA, et al. Pathological evidence of pulmonary thrombotic phenomena in severe COVID-19. J Thromb Haemost. 2020. Available from: https://doi.org/10.1111/jth.14844.

16 Yao Y, Cao J, Wang Q, Liu K, Luo Z, Yu K, et al. D-dimer as a biomarker for disease severity and mortality in COVID-19 patients: a case control study. Crit Care Emerg Med. 2020. Available from: https://doi.org/ 10.21203/rs.3.rs-20850/v1.

17 Danzi GB, Loffi M, Galeazzi G, Gherbesi E. Acute pulmonary embolism and COVID-19 pneumonia: a random association? Eur Heart J. 2020 Mar:ehaa254.

18 Xie Y, Wang X, Yang P, Zhang S. COVID-19 complicated by acute pulmonary embolism. Radiol Cardiothorac Imaging. 2020;2(2):e200067. 
19 Darzi AJ, Karam SG, Charide R, Etxeandia Ikobaltzeta I, Cushman M, Gould MK, et al. Prognostic factors for VTE and bleeding in hospitalized medical patients: a systematic review and meta-analysis. Blood. 2020 Feb: 2019003603.

20 Driggin E, Madhavan MV, Bikdeli B, Chuich T, Laracy J, Bondi-Zoccai G, et al. Cardiovascular considerations for patients, health care workers, and health systems during the coronavirus disease 2019 (COVID-19) pandemic. J Am Coll Cardiol. 2020 Mar:S0735-1097(20) 34637-4.

21 Guo T, Fan Y, Chen M, Wu X, Zhang L, He T, et al. Cardiovascular implications of fatal outcomes of patients with coronavirus disease 2019 (COVID-19). JAMA Cardiol. 2020. Available from: https://doi.org/10.1001/ jamacardio.2020.1017.

22 Shi S, Qin M, Shen B, Cai Y, Liu T, Yang F, et al. Association of cardiac injury with mortality in hospitalized patients with COVID-19 in Wuhan, China. JAMA Cardiol. 2020. Available from: https://doi.org/10.1001/jamacardio.2020.0950.

23 Johnson ED, Schell JC, Rodgers GM. The Ddimer assay. Am J Hematol. 2019 Jul;94(7): 833-9.

$24 \mathrm{Yu} \mathrm{B}, \mathrm{Li}$ X, Chen J, et al. Evaluation of variation in D-dimer levels among COVID-19 and bacterial pneumonia: a retrospective analysis. Infect Dis. 2020. Available from: https://doi. org/10.21203/rs.3.rs-20056/v1.

25 Zuckier LS, Moadel RM, Haramati LB, Freeman L. Diagnostic evaluation of pulmonary embolism during the COVID-19 pandemic. J Nucl Med. 2020 May;61(5):630-1.

26 Weg JG, Froehlich JB. Ultrasonography of leg veins in patients suspected of having pulmonary embolism. Ann Intern Med. 1998 Feb; 128(3):243.

27 Lim W, Le Gal G, Bates SM, Righini M, Haramati LB, Lang E, et al. American Society of Hematology 2018 guidelines for management of venous thromboembolism: diagnosis of venous thromboembolism. Blood Adv. 2018 Nov;2(22):3226-56.

28 Liu Y, Gayle AA, Wilder-Smith A, Rocklöv J. The reproductive number of COVID-19 is higher compared to SARS coronavirus. J Travel Med. 2020 Mar;27(2):taaa021.
29 Tritschler T, Kraaijpoel N, Le Gal G, Wells PS. Venous thromboembolism: advances in diagnosis and treatment. JAMA. 2018 Oct; 320(15):1583-94.

30 Kearon C, Akl EA, Ornelas J, Blaivas A, Jimenez D, Bounameaux H, et al. Antithrombotic therapy for VTE disease: CHEST Guideline and Expert Panel Report. Chest. 2016 Feb;149(2):315-52.

31 Wang J, Hajizadeh N, Moore EE, et al. Tissue plasminogen activator (tPA) treatment for COVID-19 associated acute respiratory distress syndrome (ARDS): a case series. J Thromb Haemost. 2020. Available from: https://doi.org/10.1111/jth.14828.

32 Phend C. Anticoagulation Guidance Emerging for Severe COVID-19 [cited 2020 Apr 9]. Infect Dis. 2020. Available from: https://www. medpagetoday.com/infectiousdisease/covid $19 / 85865$.

33 University of Liverpool. COVID-19 interactions [cited 2020 Apr 16]. Available from. https://www.covid19-druginteractions.org/.

34 Bikdeli B, Madhavan MV, Jimenez D, Chuich T, Dreyfus I, Driggin E, et al. COVID-19 and thrombotic or thromboembolic disease: implications for prevention, antithrombotic therapy, and follow-up. J Am Coll Cardiol. 2020 Apr:S0735-1097(20)35008-7.

35 Yin S, Huang M, Li D, Tang N. Difference of coagulation features between severe pneumonia induced by SARS-CoV2 and non-SARSCoV2. J Thromb Thrombolysis. 2020. Available from: https://doi.org/10.1007/s11239020-02105-8.

36 Tang N, Bai H, Chen X, Gong J, Li D, Sun Z. Anticoagulant treatment is associated with decreased mortality in severe coronavirus disease 2019 patients with coagulopathy. J Thromb Haemost. 2020. Available from: https://doi.org/10.1111/jth.14817.

37 Gao Y, Li T, Han M, et al. Diagnostic utility of clinical laboratory data determinations for patients with the severe COVID-19. J Med Virol. 2020. Available from. https://doi. org/10.1002/jmv.25770.

38 Chen T, Wu D, Chen H, Yan W, Yang D, Chen G, et al. Clinical characteristics of 113 deceased patients with coronavirus disease 2019: retrospective study. BMJ. 2020 Mar; 368:m1091
39 American Society of Hematology. COVID-19 and VTE/anticoagulation: frequently asked questions [cited 2020 Apr 16]. Available from: https://hematology.org: 443/covid-19/covid19-and-vte-anticoagulation.

40 Thachil J, Tang N, Gando S, et al. ISTH interim guidance on recognition and management of coagulopathy in COVID-19. J Thromb Haemost. 2020. Available from: https://doi.org/10.1111/jth.14810.

41 Phillippe HM. Overview of venous thromboembolism. Am J Manag Care. 2017 Dec;23(20 Suppl):S376-82.

42 Cho JK, Han JH, Park SW, Kim KS. Deep vein thrombosis after spine operation in prone position with subclavian venous catheterization: a case report. Korean J Anesthesiol. 2014 Jul; 67(1):61-5

43 Yokoyama M, Ueda W, Hirakawa M, Yamamoto $\mathrm{H}$. Hemodynamic effect of the prone position during anesthesia. Acta Anaesthesiol Scand. 1991 Nov;35(8):741-4.

44 Guo J, Huang Z, Lin L, Lv J. Coronavirus disease 2019 (COVID-19) and cardiovascular disease: a viewpoint on the potential influence of angiotensin-converting enzyme inhibitors/ angiotensin receptor blockers on onset and severity of severe acute respiratory syndrome coronavirus 2 infection. J Am Heart Assoc. 2020 Apr;9(7):e016219.

45 Phend C. COVID-19: abnormal clotting common in more severe disease [cited $2020 \mathrm{Apr}$ 9]. Available from: https://www.medpagetoday.com/infectiousdisease/covid19/85577.

46 Chen YG, Lin TY, Huang WY, Lin CL, Dai MS, Kao CH. Association between pneumococcal pneumonia and venous thromboembolism in hospitalized patients: A nationwide population-based study. Respirology. 2015 Jul;20(5):799-804.

47 Zhang Y, Xiao M, Zhang S, et al. Coagulopathy and antiphospholipid antibodies in patients with Covid-19. N Engl J Med. $2020 \mathrm{Apr}$ 23;382(17):e38.

48 Campbell CM, Kahwash R. Will complement inhibition be the new target in treating $\mathrm{CO}$ VID-19 related systemic thrombosis? Circulation. 2020. Available from: https://doi.org/ 10.1161 / C I R C ULA TION A H A. 120 . 047419 .

49 Gupta N, Zhao YY, Evans CE. The stimulation of thrombosis by hypoxia. Thromb Res. 2019 Sep;181:77-83. 